Article

\title{
Preparation and Properties of 3D Printed Alginate-Chitosan Polyion Complex Hydrogels for Tissue Engineering
}

\author{
Qiongqiong Liu ${ }^{1,2}$, Qingtao $\mathrm{Li}^{1,2}$, Sheng $\mathrm{Xu}^{2,3}$, Qiujian Zheng ${ }^{4, *}$ and Xiaodong Cao ${ }^{2,3, *}$ \\ 1 School of Medicine, South China University of Technology, 382 Outer Ring Rd, Guangzhou 510006, China; \\ 13242845287@163.com (Q.Liu); qingtaoli007@163.com or mcqtli@scut.edu.cn (Q.Li) \\ 2 National Engineering Research Center for Tissue Restoration and Reconstruction, \\ South China University of Technology, 382 Outer Ring Rd, Guangzhou 510006, China; 13662466914@163.com \\ 3 School of Material Science and Engineering, South China University of Technology, 381 Wushan Rd, \\ Guangzhou 510641, China \\ 4 Guangdong General Hospital, 106 Zhongshan Second Rd, Guangzhou 510080, China \\ * Correspondence: zqj650@126.com (Q.Z.); caoxd@scut.edu.cn (X.C.); Tel.:+86-186-7596-6509 (X.C.)
}

Received: 9 May 2018; Accepted: 9 June 2018; Published: 14 June 2018

\begin{abstract}
Three-dimensional (3D) printing holds great potential for preparing sophisticated scaffolds for tissue engineering. As a result of the shear thinning properties of an alginate solution, it is often used as 3D printing ink. However, it is difficult to prepare scaffolds with complexity structure and high fidelity, because the alginate solution has a low viscosity and alginate hydrogels prepared with $\mathrm{Ca}^{2+}$ crosslinking are mechanically weak. In this work, chitosan powders were dispersed and swelled in an alginate solution, which could effectively improve the viscosity of an alginate solution by 1.5-4 times. With the increase of chitosan content, the shape fidelity of the 3D printed alginate-chitosan polyion complex (AlCh PIC) hydrogels were improved. Scanning electron microscope (SEM) photographs showed that the lateral pore structure of 3D printed hydrogels was becoming more obvious. As a result of the increased reaction ion pairs in comparison to the alginate hydrogels that were prepared with $\mathrm{Ca}^{2+}$ crosslinking, AlCh PIC hydrogels were mechanically strong, and the compression stress of hydrogels at a 90\% strain could achieve 1.4 MPa without breaking. In addition, human adipose derived stem cells (hASCs) adhered to the 3D printed AlCh PIC hydrogels and proliferated with time, which indicated that the obtained hydrogels were biocompatible and could potentially be used as scaffolds for tissue engineering.
\end{abstract}

Keywords: 3D printed hydrogels; polyion complex; alginate; chitosan

\section{Introduction}

Three-dimensional (3D) printing and bioprinting is a rapidly growing field that aims to develop sophisticated constructs for tissue regeneration. These approaches hold the potential to achieve functional tissue constructs by repairing the complex architecture and organization of native tissues. As such, the preparation complex structure with a similar native tissue is important [1]. Hydrogels are important materials for the preparation of 3D printed tissue-engineered scaffolds [2]. The precursor solution act as 3D printing ink for preparing hydrogels. Its viscosity and transitions process of sol to gel determines the shape fidelity and structure of 3D printed hydrogels [3]. Adding nanomaterial into 3D printing ink or preparing hydrogels with double networks are the regular methods [4-6], but the biocompatibility and degradation properties of 3D printed hydrogels that are used as scaffolds for tissue engineering cannot be guaranteed [7]. For the preparation of 3D printed, hydrogels act as 
scaffolds for tissue engineering, with a complex structure and high shape fidelity, however there are still many problems that need to be solved.

Sodium alginates, which are extracted from brown seaweed, are biocompatible polyanionic [8]. As a result of the shear thinning properties of the alginate solution, it is often used as precursor solution for 3D printed tissue-engineered constructs $[9,10]$. When an alginate solution was used for preparing tissue-engineered scaffolds, the structure and shape fidelity of the 3D printed hydrogels is usually difficult to guarantee, because the viscosity of the maximum concentration of alginate solution is still insufficient, the deposited filaments are easily fused and collapsed. In addition, the alginate hydrogels that are crosslinked with $\mathrm{Ca}^{2+}$ are mechanically weak [4], and the deposited filaments are easily collapsed because of gravity, which also has influence on the structure and shape fidelity of 3D printed hydrogels.

Acting as a biocompatible polyanionic, the alginate can also be crosslinked with polycation to obtain polyion complex (PIC). The mechanical strength of PIC hydrogels, prepared with two oppositely charged polyelectrolytes, is controllable through changing reactive ion pairs [11]. The mixing of bulk solutions of polycation and polyanion usually leads to inhomogeneous precipitation, where a strong PIC is formed at the interface of the two solutions, which quenches the further reaction [11,12]. As a result of the limited reactive ion pairs that are involved in the reaction, the crosslinking density of the biomaterial internal network is low. To solve this problem, Luo et al. [13] polymerized one of the polyelectrolytes from its monomers solution in the presence of another oppositely charged polymer at 1:1 charge ratio. The cationic monomer 3-(methacryloylamino) propyl-trimethylammonium chloride (MPTC) was homopolymerized in the first step and was then mixed with the anionic monomer sodium p-styrenesulfonate (NaSS). After the well dispersion, the anionic monomer is polymerized in the second step, to form soft PIC hydrogel. Tensile and compression tests indicate that the hydrogels that are formed by the oppositely charged polyelectrolytes are tough, self-healing, and rebuildable.

Chitosan is the only natural polycation polysaccharide, which is swelling, but it is insoluble in an aqueous solution. When the $\mathrm{pH}$ value of the aqueous solution is less than 4 , chitosan is soluble and can react with anion or polyanion, such as alginate, to prepare the PIC hydrogels [14]. In this work, chitosan powders were added into the alginate solution as the 3D printing ink, and chitosan is swelling but insoluble in the alginate solution, which can effectively improve the viscosity of the alginate solution. After being treated with a hydrochloric acid $(\mathrm{HCl})$ solution, the chitosan powders that were dispersed in am alginate solution were soluble and reacted with alginate, thus, 3D printed of AlCh PIC hydrogels were obtained. A molecule of $\mathrm{HCl}$ is a small molecule that diffuses easily, thus, the further reaction between two oppositely charged polyelectrolytes will not be quenched. With the increase of the reactive ion pairs, the mechanical strength of the 3D printed AlCh PIC hydrogel can be improved, and the 3D printed hydrogels with complex structures could also be prepared correspondingly.

\section{Materials and Methods}

\subsection{Materials}

Sodium alginate ( $\mathrm{Al}$, viscosity: 180-220 mp.s) and chitosan (Ch, viscosity: 100-200 mp.s, and the degree of deacetylation $>95 \%$ ) were both obtained from Aladdin. $\mathrm{HCl}$ was obtained from Huachengda Chemical Co. Ltd. (Zhuhai, China). All off the reagents were used as they were received if there was no special explanation.

\subsection{Preparation of $3 D$ Printing Ink}

The 3D printing ink was prepared as two steps. Firstly, a 10\% (w/v) alginate solution was prepared by adding sodium alginate into deionized water (DI water) at room temperature. Secondly, a certain amount of chitosan (as seen in Table 1) was added to the alginate solution and stirred sufficiently to ensure a good dispersion of chitosan in the alginate solution. To remove the air bubbles in the $3 \mathrm{D}$ printing ink, it was placed in vacuum drying oven at $30^{\circ} \mathrm{C}$ for $30 \mathrm{~min}$. 
Table 1. Three-dimensional (3D) printing ink made from different molar ratio of alginate to chitosan.

\begin{tabular}{ccccc}
\hline 3D Printing Ink & Alginate $(\mathbf{g})$ & Chitosan $(\mathbf{g})$ & Water $(\mathbf{m L})$ & Alginate:Chitosan $(\mathbf{m o l}: \mathbf{m o l})$ \\
\hline Al1Ch0 & 2 & 0 & 20 & $1: 0$ \\
Al1Ch0.2 & 2 & 0.333 & 20 & $1: 0.2$ \\
Al1Ch0.4 & 2 & 0.665 & 20 & $1: 0.4$ \\
Al1Ch0.6 & 2 & 0.998 & 20 & $1: 0.6$ \\
Al1Ch0.8 & 2 & 1.331 & 20 & $1: 0.8$ \\
Al1Ch0.9 & 2 & 1.497 & 20 & $1: 0.9$ \\
Al1Ch1.0 & 2 & 1.663 & 20 & $1: 1.0$ \\
Al1Ch1.1 & 2 & 1.830 & 20 & $1: 1.1$ \\
Al1Ch1.2 & 2 & 1.996 & 20 & $1: 1.2$ \\
\hline
\end{tabular}

\subsection{Preparation of $3 D$ Printed Hydrogels}

The 3D-BIOPLOTTER ${ }^{\mathrm{TM}}$ (Envisionter Gmbh, Karlsruhe, Germany) with a resolution of $100 \mu \mathrm{m}$ was used for the printing of all of the types of hydrogels. The 3D printing ink (Al1Ch0.2-Al1Ch1.2) was loaded into a dispensing unit consisting of a cartridge and a nozzle $(400 \mu \mathrm{m})$, and was extruded out with applied nitrogen gas along the $\mathrm{X}-\mathrm{Y}-\mathrm{Z}$ target paths. The spacing between the two deposited fibers was set as $900 \mu \mathrm{m}$. After a layer of deposition was completed, the staying between the layers was $15 \mathrm{~s}$. In the meantime, an $\mathrm{HCl}$ solution $(0.5 \mathrm{~mol} / \mathrm{L})$ was sprayed on the deposited fibers with a single-use syringe, in order to protonate the $\mathrm{NH}_{2}$ on the chitosan. As the amino group was converted to ammonium, the 3D printed alginate-chitosan polyion complex hydrogels were prepared because the electrostatic interaction between the two oppositely charged polyelectrolytes (as shown in Figure 1). To verify the effects of the addition of chitosan on the morphology of, 3D printed alginate hydrogels were prepared in the same ways, except that the $\mathrm{HCl}$ solution was replaced with a $\mathrm{CaCl}_{2}$ solution $(0.1 \mathrm{~mol} / \mathrm{L})$. If it was not specifically stated, the angle of layers of all of the hydrogels that were used for the characterization was $90^{\circ}$.

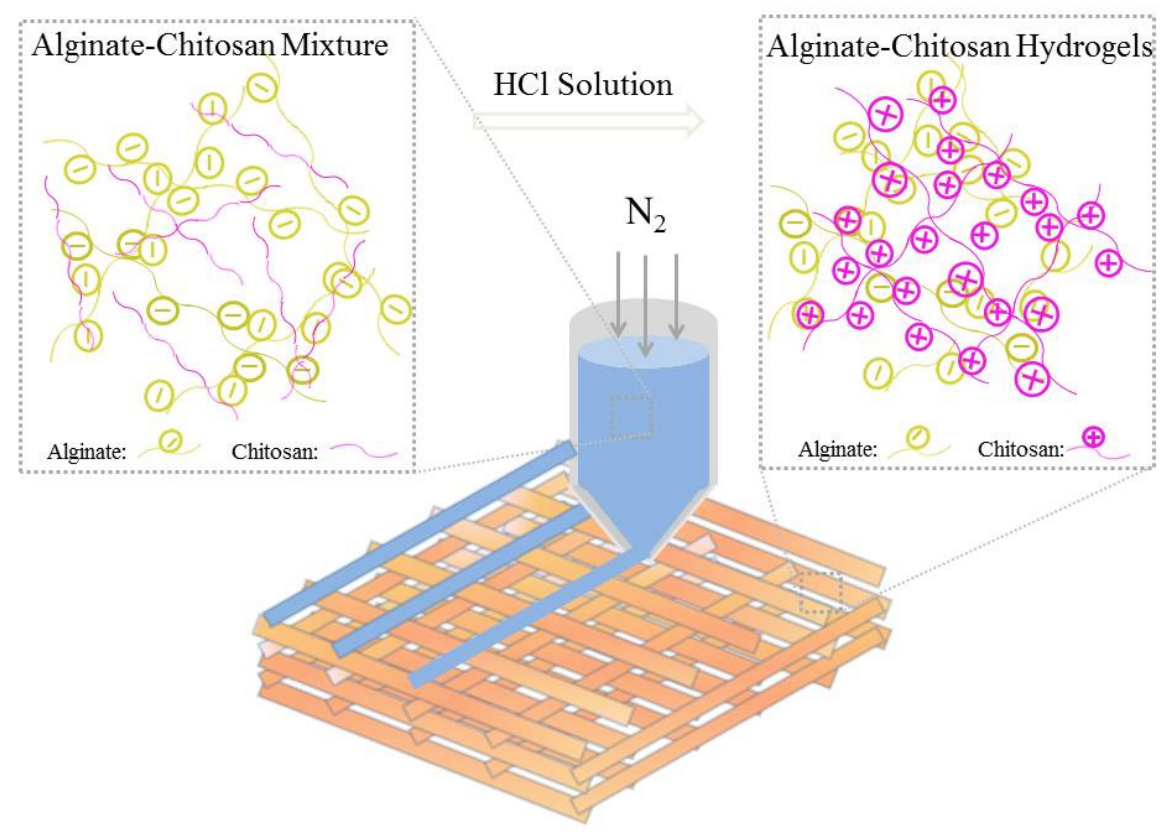

Figure 1. Schematic diagram of three-dimensional (3D) printing alginate-chitosan polyion complex hydrogels.

\subsection{Rheological Test of 3D Printing Ink}

Rheological properties of 3D printing ink with a different molar ratio of alginate to chitosan were measured on a rotary rheometer (AR-G2, TA, Waters, Newcastle, DE, USA), with parallel circular 
plates of a $40 \mathrm{~mm}$ diameter. Steady rate sweeps were conducted by varying the shear rates from 0.01 to $1000 \mathrm{~s}^{-1}$ at $30{ }^{\circ} \mathrm{C}$, and the viscosity was measured at different shear rates.

\subsection{Fourier-Transform Infrared Spectroscopy}

A Fourier-transform infrared (FTIR) spectroscopy (Nexus Por Euro, Bruker, Karlsruhe, Germany) was used to verify the electrostatic interaction between alginate and chitosan. Using the FTIR spectra of alginate, chitosan, and 3D printed, the AlCh PIC hydrogel was obtained under the following conditions: the average of 32 scans between $400 \mathrm{~cm}^{-1}$ and $4000 \mathrm{~cm}^{-1}$ at a resolution of $4 \mathrm{~cm}^{-1}$.

\subsection{Scanning Electron Microscope (SEM) Analysis}

The structure and architecture of the 3D printed hydrogels was observed using an SEM (Quanta 200, FEI, Eindhoven, The Netherlands). The 3D printed hydrogels were prefrozen at $-20{ }^{\circ} \mathrm{C}$ in a refrigerator and then freeze-dried in a freeze-drying machine (VIRTIS Genesis, Warminster, PA, USA). Before being mounted on aluminum stubs, they were treated with gold sputtering. The front and side morphology of the freeze-dried hydrogels were examined under SEM at an accelerated voltage of $10 \mathrm{KV}$, and the work distance (WD) was about $10 \mathrm{~mm}$, which was adjusted according to the sample height.

\subsection{Mechanical Properties}

Compression tests of the rectangular block hydrogels with a size $10 \mathrm{~mm} \times 10 \mathrm{~mm} \times 3.2 \mathrm{~mm}$ were conducted on a universal material testing machine (Instron 5967, Instron, Norwood, MA, USA). The compression stress-strain curves were obtained when hydrogels were uniaxially compressed at a displacement rate of $1 \mathrm{~mm} / \mathrm{min}$ to $90 \%$ strain. The compression strength and toughness were calculated through stress-strain curves. Compression stress with $90 \%$ strain represented the compression strength, while the toughness was equal to the area under the stress-strain curves. The compression modulus was calculated from the slope of the linear elastic region of the stress-strain curves, which was between $0 \%$ and $30 \%$ strain for all of the samples. In order to get the mean and standard deviation calculations, five parallel samples of each group were used for testing.

\subsection{Swelling Ratio and Water Absorption of Hydrogels}

For the swelling ratio and water absorption, rectangular block hydrogels of different internal structures, in size of $10 \mathrm{~mm} \times 10 \mathrm{~mm} \times 3.2 \mathrm{~mm}$, were measured according to the reported methods by the authors of [15]. The 3D printed hydrogels were prefrozen at $-20^{\circ} \mathrm{C}$ in a refrigerator and then freeze-dried in a freeze-drying machine (VIRTIS Genesis, Warminster, PA, USA) to obtain freeze-dried hydrogels. The weight of the freeze-dried hydrogels was denoted as $W_{0}$, and after that, they were immersed in phosphate buffered saline (PBS, $\mathrm{pH}=7.4$ ) at the $37^{\circ} \mathrm{C}$ constant temperature using a shake table. At a fixed time, the PBS on the scaffold surface was wiped off, and the weight of hydrogels at the fixed time was denoted as W. In order to get the mean and standard deviation calculations, five parallel samples of each group were used for testing. The swelling ratio and water absorption of the hydrogels in equilibrium were calculated as the following:

$$
\begin{gathered}
\text { Swelling ratio }=\left(\mathrm{W}-\mathrm{W}_{0}\right) / \mathrm{W}_{0} \times 100 \% \\
\text { Water absorption }=\left(\mathrm{W}-\mathrm{W}_{0}\right) / \mathrm{W} \times 100 \%
\end{gathered}
$$

\subsection{Degradation Properties of Hydrogels}

Rectangular block hydrogels, with size $10 \mathrm{~mm} \times 10 \mathrm{~mm} \times 3.2 \mathrm{~mm}$, were pre-weighted and immersed in PBS at $37^{\circ} \mathrm{C}$. At different time intervals, thee samples were taken out and rinsed with deionized water to remove the extra PBS $(\mathrm{pH}=7.4)$ on the surface of the samples, and then, they were freeze dried and weighed again. The PBS solution was changed every 3 days. In order to get the 
mean and standard deviation calculations, five parallel samples of each group were used for testing. The degradation mass ratio was calculated as the following formula:

$$
\text { Degradation mass ratio }=\left(\mathrm{W}_{1}-\mathrm{W}_{2}\right) / \mathrm{W}_{1} \times 100 \%
$$

where $W_{1}$ is the initial weight of the hydrogel and $W_{2}$ is final weight of the hydrogel.

\subsection{Cell Viability Assay and Cell Morphology}

Human adipose-derived stem cells (hASCs) were purchased from Cyagen Biosciences (Guangzhou, China) and were incubated to passage 5-10 in a culture medium (DMEM (dulbecco's modified eagle medium) consisting of $10 \%$ FBS, (fetal bovine serum) $1 \%$ penicillin/streptomycin) at $37{ }^{\circ} \mathrm{C}$ in a constant temperature incubator. To seed the cells on the 3D printed AlCh PIC hydrogels, there were three steps. Firstly, the Al1Ch1.0 hydrogels, with size $10 \mathrm{~mm} \times 10 \mathrm{~mm} \times 2 \mathrm{~mm}$, were freeze-dried with a freeze-dring machine and were placed in the 24-well plates to be sterilized by $15 \mathrm{KGy} \gamma$ radiation. Secondly, the cell culture medium was added into the pores to immerse the dried hydrogels for $24 \mathrm{~h}$ in a super clean table. Thirdly, the cell culture medium was taken out, the material was air-dried in a super clean table for $30 \mathrm{~min}$, and the cultured cells were dissociated with $0.25 \%$ trypsin-EDTA and centrifuged, and then, hASCs in $100 \mu \mathrm{L}$ complete medium were seeded on each sample and incubated at $37^{\circ} \mathrm{C}$ in a constant temperature incubator for $1 \mathrm{~h}$. After this, $900 \mu \mathrm{L}$ of the complete medium was added to each sample so as to give a cell density of $2 \times 10^{5}$ cells per mL. The complete medium was changed every 2 days.

The cell viability of the constructs was examined by a Live-Dead viability Kit after the cells were cultured for 3 days. For the Live-Dead assay, firstly, the cell-laden hydrogels were washed with sterilized PBS 3 times; secondly, $100 \mu \mathrm{L}$ of the live-dying stain was added to the pores; thirdly, 24-well plates were incubated at the $37^{\circ} \mathrm{C}$ in a constant temperature incubator for $30 \mathrm{~min}$; and finally, they were washed with sterilized PBS $(\mathrm{pH}=7.4) 3$ times. With the aid of a fluorescence inverted microscope, the live and dead cells that were distributed on the hydrogels were observed.

The morphology and adhesive properties of hASCs in the 3D printed hydrogels were observed by SEM (Quanta 200, FEI, Eindhoven, the Netherlands) after the cells were cultured for 3 days. The cells were fixed by glutaraldehyde $(2.5 \%, v / v)$ overnight, after being washed with PBS, and they were dehydrated with an isocratic gradient ethanol solution. Then, they were dried in an air oven at $37^{\circ} \mathrm{C}$ for $3 \mathrm{~h}$ and the morphology and adhesive properties of the hASCs were observed.

\subsection{Proliferation of hASCs Cultured on Materials}

The hASCs were incubated to passage 5-10 in a culture medium (DMEM consisting of $10 \%$ FBS, $1 \%$ penicillin/streptomycin) at $37^{\circ} \mathrm{C}$ in a constant temperature incubator. Then, $2 \times 10^{5} / \mathrm{mL}$ of hASCs in $100 \mu \mathrm{L}$ were seeded on the sterilized Al1Ch1.0 hydrogels, with size $10 \mathrm{~mm} \times 10 \mathrm{~mm} \times 2 \mathrm{~mm}$, and $1 \mathrm{~h}$ later, $900 \mu \mathrm{L}$ of a culture medium was added to each sample, in order to give a cell density of $2 \times 10^{5}$ cells per mL. Samples were taken out to place in another 24-well plate at fixed time, the proliferation of the hASCs were evaluated with a Cell Counting Kit-8 (CCK-8, Dojindo, Kumamoto, Japan), according to the manufacturer's instruction. There was $1 \mathrm{~mL}$ of CCK-8 solution added to pores after hhe cells were incubated for $1 \mathrm{~h}$ at $37^{\circ} \mathrm{C}$ in a constant temperature incubator. Finally, the optical density of the CCK-8 solution was measured at $450 \mathrm{~nm}$ using a microplate reader (Therm 3001, Ithaca, NY, USA). In order to get the mean and standard deviation calculations, five pores were used for testing.

\subsection{Statistical Analysis}

The data were expressed as mean \pm standard deviation (SD). The statistical analysis was performed using the one way ANOVA (analysis of variance) test to determine significant differences. A $p$-value $<0.05$ was considered as statistically significant. 


\section{Results and Discussion}

\subsection{Rheology of 3D Printing Ink}

Rheology is the study of the flow of polymer solution under the application of shear stress. The study of the rheology properties of 3D printing ink is important for the 3D printed process. A high viscosity impedes both the droplet formation, resulting from surface tension force, and the collapse of deposited filaments. The shear thinning of the polymer solution is caused by shear-induced conformation changes in molecular chains from curly to stretched, which determines the continuous extrusion of 3D printing ink from the needle [2]. To study the effect of the content of chitosan on the viscosity of 3D printing ink, rheology tests of 3D printing ink were performed and the results are shown in Figure 2. When the concentration of the alginate solution increased to $10 \%(w / v)$, its further dissolution became more difficult, and the viscosity of the alginate solution could not be improved. However, a viscosity of $10 \%(w / v)$ in an alginate solution was insufficient for preparing hydrogels with complex structures, because of the collapse of deposited filaments. The chitosan was swelling but insoluble in alginate solution, in consequence, the viscosity of 3D printing ink prepared by adding chitosan powders into alginate solution can be improved. With the increased content of chitosan, Al1Ch1.2 had a viscosity of $3627 \mathrm{~Pa} \cdot \mathrm{s}$, about four times that of Al1Ch0. As with the rheological test results of the sodium alginate solution, the viscosity of the 3D printing ink that was prepared by the addition of chitosan powders into an alginate solution decreased with the increased shear force. Therefore, an addition of chitosan into an alginate solution was effective for improving the viscosity of 3D printing ink, and had the potential to prepare hydrogels with complex structures.

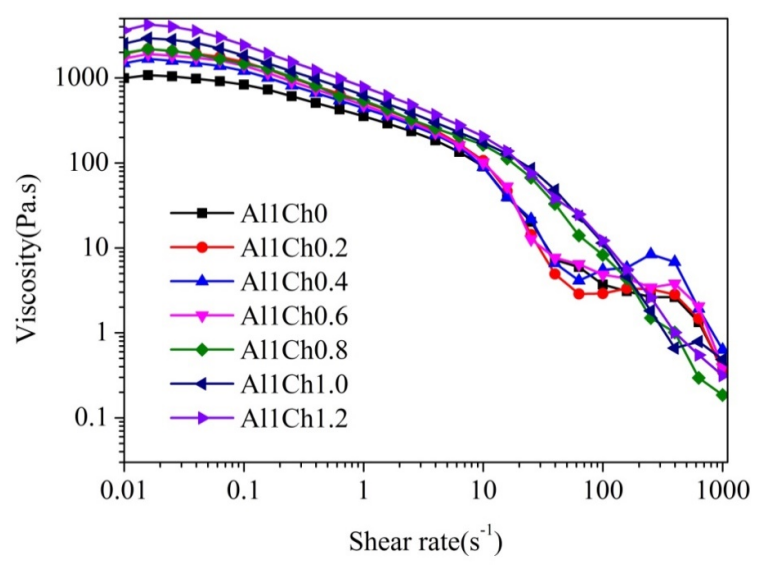

Figure 2. Rheology of 3D printing ink.

\subsection{Morphology of 3D Printed Hydrogels}

The viscosity of 3D printing ink had an important influence on the structure of 3D printed of hydrogels. Because a high viscosity impeded the collapse of deposited filaments, the lateral pores of the 3D printed hydrogels were intact, and the height of hydrogels could also be larger than hydrogels that were prepared with low viscosity ink. To study the effect of 3D printing ink on the structure of 3D printed hydrogels, morphologies of freeze-dried hydrogels that were prepared with different 3D printing inks are shown in Figure 3. As a result of the relatively low viscosity of the $10 \%(w / v)$ alginate solution, the deposited filaments were easily collapsed, and the height of the 3D printed alginate hydrogel was difficult to be larger than $2 \mathrm{~mm}$, which is seen in Figure 3(a2). As for the AlCh PIC hydrogels, as the chitosan swelled in the alginate solution, the increased viscosity was useful for preparing complex 3D printed hydrogels. The structure of hydrogels was improving with the increased content of chitosan in the 3D printing ink, as shown in Figure 3(b1-e2). The height of the 3D printed AlCh PIC hydrogels that were prepared with Al1Ch0.2-Al1Ch0.4 was difficult to be larger than $2 \mathrm{~mm}$, moreover, the lateral 
pores were not obvious because of the collapse of the deposited filaments. However, the height of the 3D printed AlCh PIC hydrogels that were prepared with Al1Ch0.6-Al1Ch0.8 was getting larger than $3.5 \mathrm{~mm}$. 3D printed hydrogels with a complex structure and high shape fidelity could be prepared when the molar ratio of chitosan to alginate was larger than 0.8 .

To verify that the 3D printing ink, which was prepared by adding a sufficient amount of chitosan into am alginate solution, was useful for printing hydrogels with complex structures, a nose model was built, after that, a 3D printed nose with a height of $20 \mathrm{~mm}$ was printed with Al1Ch1.2 printing ink (as seen in Figure 4b). From the photo of the 3D printed nose, it was consistent with the nose model that was used for 3D printed and had no collapse. From the 3D printed nose, we proved that the prepared 3D printing ink could be used to print organs with complex structures.

Under the condition of the same distance between adjacent filaments, the angle of filaments between layers led to the decrease of pore diameter, and thus, the hydrogels with different internal structures could be printed by changing the angle of the filaments between the layers. With the Al1Ch1.0 printing ink, we prepared the hydrogels with different internal structures by changing the angles between the layers, under the condition that the distance between the adjacent fibers was $0.9 \mathrm{~mm}$ (as shown in Figure 4(c1-e2)).
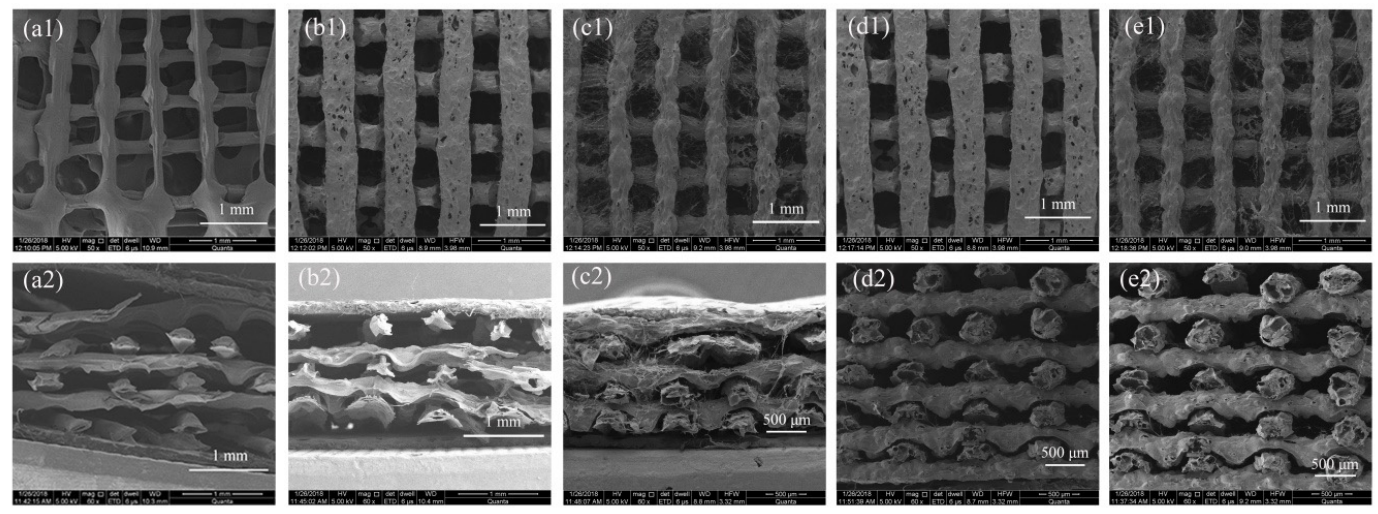

Figure 3. Morphological characterization of 3D printed hydrogels. Scanning electron microscope (SEM) micrographs of hydrogel prepared with 3D printing ink: (a1,a2) Al1Ch0; (b1,b2) Al1Ch0.2; (c1,c2) Al1Ch0.4; (d1,d2) Al1Ch0.6; and (e1,e2) Al1Ch0.8 (1 and 2 represent the front and side, respectively, of the $3 \mathrm{D}$ printed hydrogels).
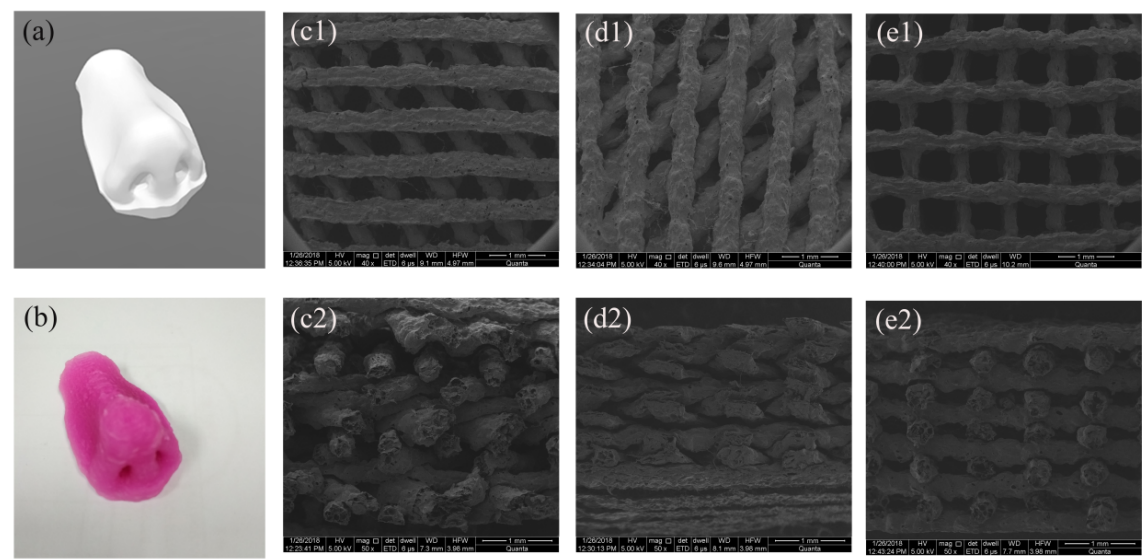

Figure 4. Morphological characterization of 3D printed hydrogels. Photographs of (a) 3D model of nose and (b) 3D printed nose prepared with 3D printing ink Al1Ch1.2. SEM micrographs of hydrogel with different angles between filaments, prepared with 3D printing ink Al1Ch1.0: (c1,c2) 45 ; (d1,d2) $60^{\circ} ;(\mathbf{e} 1, \mathbf{e} 2) 90^{\circ}$ (1 and 2 represent the front and side, respectively, of the scaffolds). 


\subsection{Fourier Transform Infrared Spectroscopy}

To verify that the 3D printed AlCh PIC hydrogels were formed by electrostatic interaction between two polyelectrolytes, the infrared absorption spectrum of alginate, chitosan, and the 3D printed $\mathrm{AlCh}$ PIC hydrogels (as shown in Figure 5) were analyzed. The characteristic peaks of the alginate were the peaks at $1604 \mathrm{~cm}^{-1}\left(\mathrm{C}=\mathrm{O}\right.$ bond) and $1422 \mathrm{~cm}^{-1}$. The characteristic peaks of chitosan were the overlapped peaks at $1666 \mathrm{~cm}^{-1}$ (amide-I) and $1596 \mathrm{~cm}^{-1}$ (amide-II). The double amide peaks in the spectrum of chitosan corresponded to the partial N-deacetylation of the chitin [16]. In the spectrum of the AlCh PIC hydrogels, the amide-I peaks shifted from $1666 \mathrm{~cm}^{-1}$ to $1728 \mathrm{~cm}^{-1}$, while the amide-peaks were significantly intensified. On the other hand, the amino peak at $1160 \mathrm{~cm}^{-1}$ and the carboxyl peak at $1604 \mathrm{~cm}^{-1}$ disappeared in the spectrum of the AlCh PIC hydrogel, which all suggested the electrostatic interaction between alginate and chitosan. Our results were similar to other authors' work $[17,18]$, who had prepared scaffolds by an electrostatic interaction between alginate and chitosan.

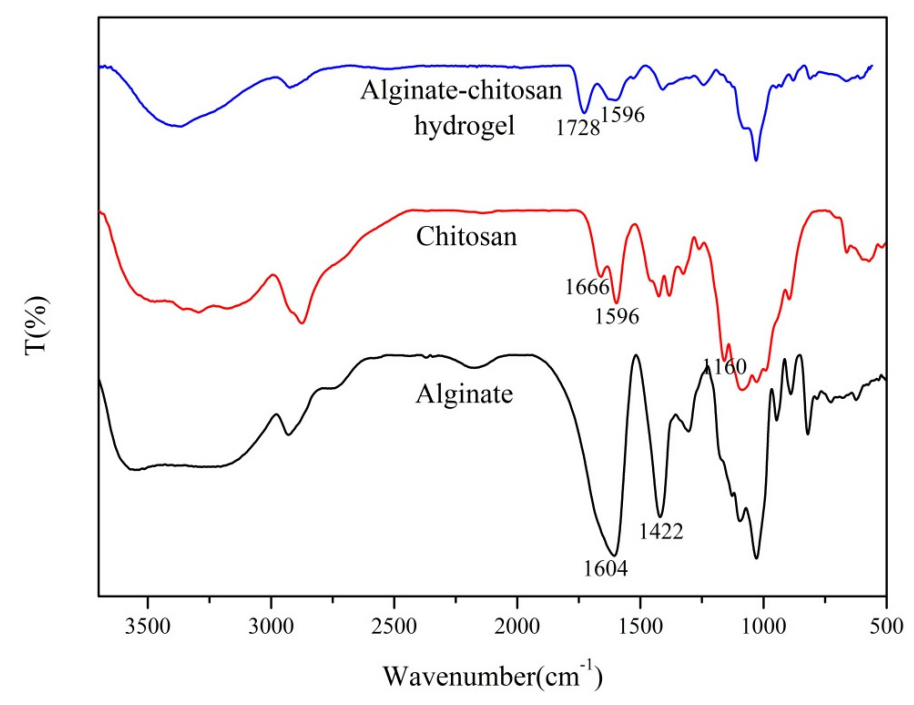

Figure 5. Fourier-transform infrared (FTIR) spectra of alginate and chitosan, and the 3D printed alginate-chitosan polyion complex hydrogels.

\subsection{Mechanical Properties of 3D Printed AlCh PIC Hydrogels}

The mixing of bulk solutions of polycation and polyanion usually led to inhomogeneous precipitation, where strong polyion complexes (PIC) were formed at the interface of the two solutions, which quenched the further reaction. Because of the limited reactive ion pairs that were involved in the reaction, the crosslinking density of the biomaterial internal network was low, and so the mechanical properties of the biomaterial were made by adding the chitosan powders into an alginate solution, and then the alginate-chitosan mixture was treated with an $\mathrm{HCl}$ solution. The changes in $\mathrm{pH}$ were used to induce a poly-complexation of the two polyelectrolytes. A molecule of $\mathrm{HCl}$ was a small molecule that diffused easily, thus, the further reaction between the two oppositely charged polyelectrolytes would not be quenched. With the increase of the reactive ion pairs, the mechanical strength of the 3D printed AlCh PIC hydrogels could be improved. At a 10\% $(w / v)$ concentration of the alginate solution, the content of chitosan had an effect on reactive ion pairs, and thus affected the mechanical properties of the 3D printed AlCh PIC hydrogels. To explore the effect of the chitosan content on the mechanical properties of 3D printedg AlCh PIC hydrogels, compression tests were conducted towards the 3D printed AlCh PIC hydrogels with different molar ratios of chitosan to alginate, and the results are shown in Figure 6. There was no significant difference in the mechanical properties between the hydrogels whose ratio of chitosan to alginate was $0.9-1.1$, but the mechanical properties of Al1Ch1.2 became poor and had a significant difference with the hydrogels that were 
prepared with the Al1Ch0.9-Al1Ch1.1 ink. When the molar ratio of chitosan to alginate was 0.9-1.1, the number of positive and negative charges that were involved in the reaction was basically the same. When the content of the chitosan increased further, the electrostatic repulsion between the excessive positive charges would affect the electrostatic interaction between opposite charges, thus leading to the decrease of the mechanical properties of the 3D printed hydrogels. For 3D printed AlCh PIC hydrogels, the maximum compression stress at $90 \%$ strain, compression modulus, and toughness could reach 1.4 MPa, $0.2 \mathrm{MPa}$, and $1.8 \times 10^{5} \mathrm{~J} / \mathrm{m}^{3}$, respectively.

When the compression tests were completed at $90 \%$ strain, the hydrogels were not broken. After being soaked in deionized water for $1 \mathrm{~h}$, the hydrogels were taken out for the second compression. The two compression stress-strain curves and modulus are shown in Figure 6e,f. There was no significant difference between the two compression modulus, which indicated that 3D printed AlCh PIC hydrogels had a good fatigue resistance.

The compression strength of 3D printed hydrogels (Al1Ch0.9) that were prepared by crosslinking $10 \%$ alginate and $7 \%$ chitosan, could achieve $1.5 \mathrm{MPa}$, which was about the same as the bulk hydrogel that were prepared by photo crosslinking 20\% PNAGA ( $N$-acryloyl glycinamide), and the much larger than bulk and 3D printed hydrogels that were prepared by adding $7 \%$ clay into a 10\% PNAGA photo crosslinking network [19]. The compression modulus of the hydrated scaffolds that were prepared by alginate and chitosan were $83.1 \pm 14.6 \mathrm{KPa}$ [20], which was far lower than the 3D printed hydrogels that were prepared by alginate and chitosan.
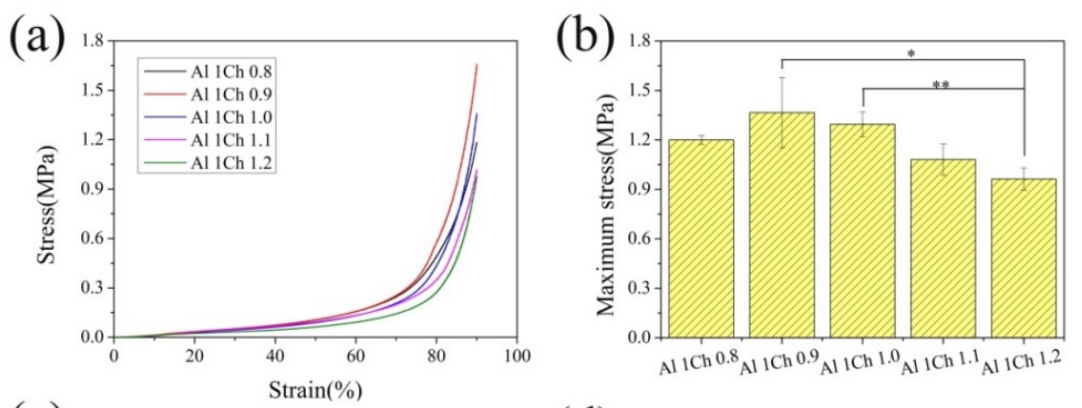

(c)

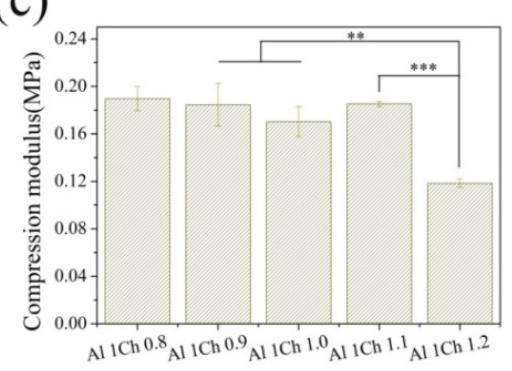

(d)
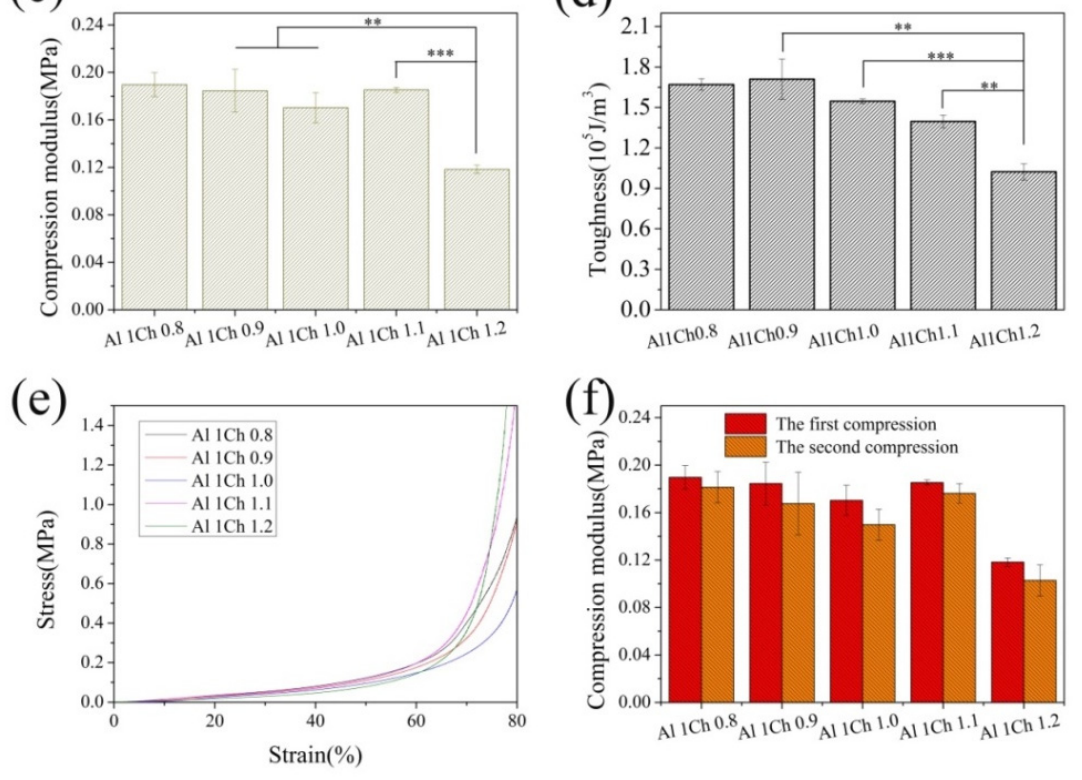

Figure 6. Compressive properties of $3 \mathrm{D}$ printed alginate-chitosan polyion complex (AlCh PIC) hydrogels prepared with different molar ratio of alginate to chitosan. The (a) compression stress-strain curves; (b) maximum stress at 90\% strain; (c) compression modulus; (d) toughness; (e) compression stress-strain curves; and (f) compression modulus of the two compressions. 


\subsection{Swelling Ratio and Water Absorption of Hydrogels}

As tissue engineering scaffolds, the swelling of the hydrogels helped to provide nutrients and promote the metabolism during the cell culture. The swelling process was greatly influenced by the internal pore structure of the 3D printed hydrogels. We prepared Al1Ch1.0 hydrogels with different internal structures, by changing the angle of layers, and then, the swelling ratio of the hydrogels with different internal structures at each time point were tested (as seen in Figure 7a). The swelling ratio and water absorption rate in the equilibrium state of swelling were concluded and, as shown in Figure $7 b, c$, all of the hydrogels had a good swell behavior. The swelling ratio of the 3D printed hydrogels with different internal structures were different, because the swelling ratio was affected by the porosity of the hydrogel, while the porosity of the 3D printed hydrogels was affected by the angle of the layers. When the angle of the layers was $90^{\circ}$, the porosity of the 3D printed AlCh PIC hydrogels was maximum as well as for the swelling ratio, and they had a significant difference with swelling ratio of the hydrogels whose angle of layers were $45^{\circ}$ and $60^{\circ}$. The maximum amount of absorbed water was 15 times the dry weight of the hydrogels. There was no significant difference between the water absorption rate of the hydrogels with a different internal structure (as shown in Figure 7c), as water accounted for most of the weight of all of the hydrogels in the equilibrium state of swelling.

(a)

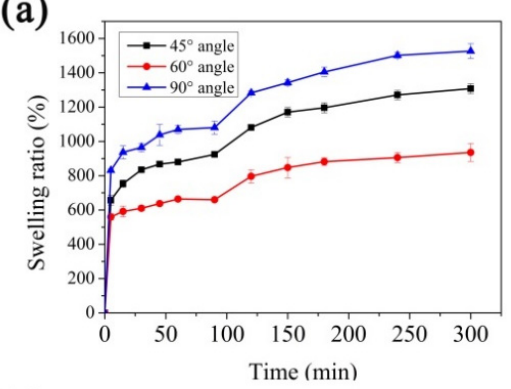

(c)

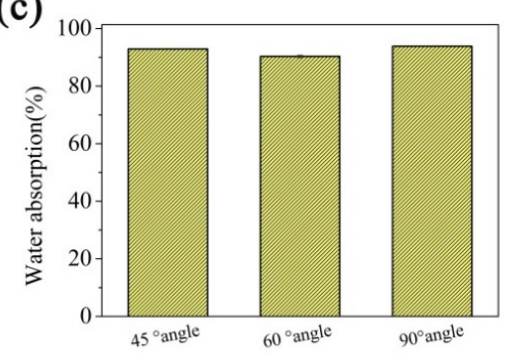

(b)

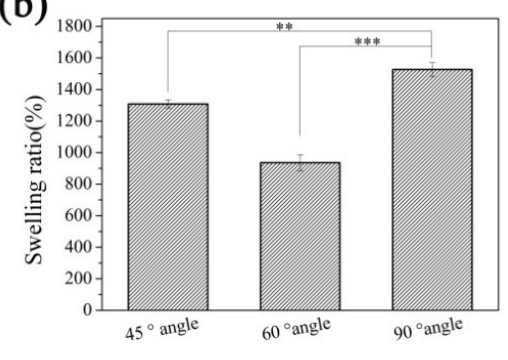

(d)

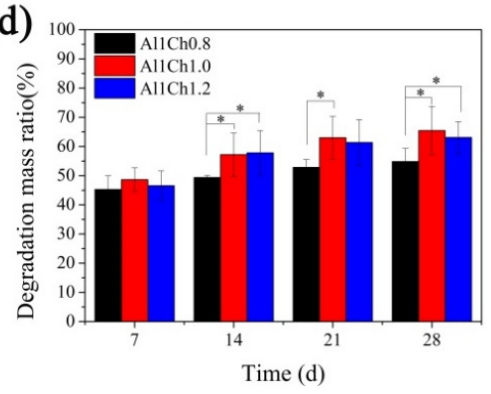

Figure 7. Physiochemical properties of 3D printed AlCh PIC hydrogels. (a) swelling process; (b) swelling ratio; (c) water absorption; (d) degradation mass ratio of hydrogels.

\subsection{Degradation Properties of Hydrogels}

A suitable degradation rate was important for the tissue engineering scaffolds, when the degradation was too fast, the scaffolds lost their role of supporting for the cells. In contrast, when the degradation rate ws too slow, it would have impeded the emergence of the neo tissue. When alginate was crosslinked through $\mathrm{Ca}^{2+}$, calcium ions were easily replaced by monovalent cation, and the hydrogels in vivo could not exist stably. However, when the alginate was crosslinked with chitosan, because of the entanglement between the molecular chains of alginate and chitosan, the crosslink networks were more stable, so the AlCh PIC could exist for a long time. Furthermore, through adjusting the ratio of the alginate to chitosan, we had a control over the degradation rate of the hydrogels. Figure $7 \mathrm{~d}$ shows the degradation process of the 3D printed AlCh PIC hydrogels. As time went on, the dry mass of the hydrogels decreased, and the degradation mass ratio of the different hydrogels at 28 days was $50-70 \%$. The content of the chitosan had an effect on the degradation 
properties because of the different crosslink density of the hydrogels. There were significant differences between the degradation mass ratios of the different hydrogels. On the other hand, we measured the mechanical modulus change of the bulk alginate hydrogel that was crosslinked with $\mathrm{Ca}^{2+}$ and the bulk AlCh PIC hydrogels, and it could be seen obviously that the AlCh PIC was more stable than the alginate hydrogels that were crosslinked with $\mathrm{Ca}^{2+}$ (seen in Figure S1). On the 12th day, the alginate hydrogel that was crosslinked with $\mathrm{Ca}^{2+}$ degraded absolutely, while the AlCh PIC hydrogels were still tough, the compression stress of the AlCh PIC hydrogels was 0.3-0.4 MPa by the 16th day.

\subsection{Biocompatibility of Hydrogels}

Stem cells are pluripotent cells, and human adipose-derived stem cells (hASCs) were obtained from adipose tissue. The use of hASCs was feasible, as the adipose tissue was easily available. Furthermore, the hASCs expressed the biochemical profile of adipocytes, chondrocytes, and osteoblasts under the appropriate conditions [21]. The hASCs were used to test the biocompatibility of the hydrogels. Figure $8 \mathrm{a}-\mathrm{c}$ shows the live-dead cells that were distributed on the hydrogels on the third day. It could be seen that the live cells spread uniformly on the hydrogels, while little or no dead cells existed, which showed the 3D printed AlCh PIC hydrogels' biocompatibility. On the other hand, the hASCs that were distributed on the materials proliferated with time, which was shown in Figure $8 \mathrm{~d}$. Figure 8e,f shows the morphology of the cells on the hydrogels on the third day, where the cells adhered to the 3D printed AlCh PIC hydrogels, and expanded along the direction of the deposited filaments.
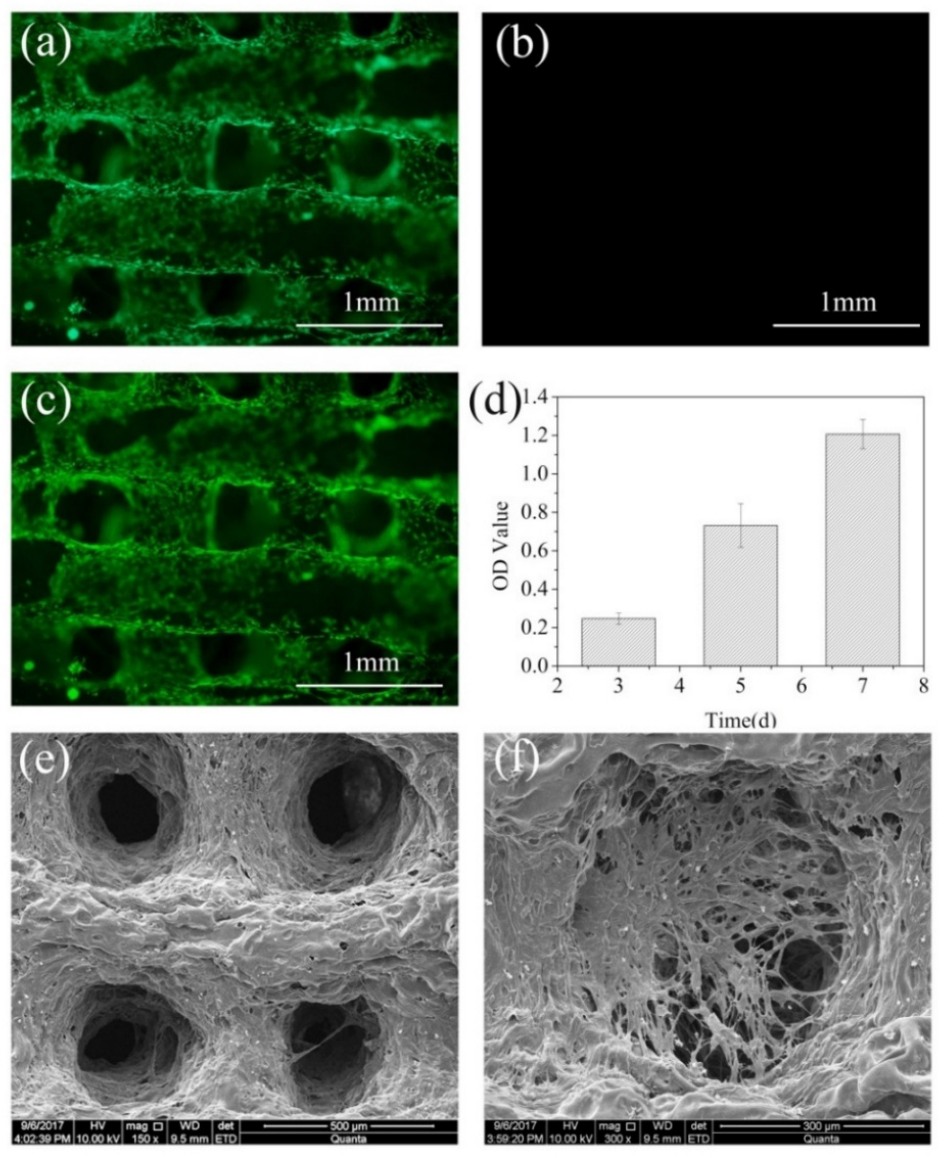

Figure 8. Biocompatibility of 3D printed AlCh PIC hydrogels. Inverted fluorescence microscope of (a) live; (b) dead; (c) merged cells at the 3rd day. (d) Proliferation of hASCs cultured on 3D printed AlCh PIC hydrogels. (e,f) SEM micrographs of 3D printed AlCh PIC hydrogels after cultured with hASCs for 3 days. 


\section{Conclusions}

In this study, we prepared 3D printed hydrogels by changing the $\mathrm{pH}$ so as to induce the poly-complexation of the alginate and chitosan after the 3D printing ink was extruded out. The addition of chitosan into the alginate solution ws useful for improving the viscosity of 3D printing ink. Moreover, the molecule of $\mathrm{HCl}$ was a small molecule that diffused easily, and the gelation process, through the change of $\mathrm{pH}$ to induce poly-complexation between the alginate and chitosan, was fast. Thus, the deposited filaments were not easily collapsed or fused, and complex constructs could be prepared through this method. The physicochemical properties of the obtained hydrogels could be controlled by controlling the content of chitosan. By printing the nose with the alginate-chitosan mixture, we proved that the prepared 3D printing ink could be used to print tissues or organs with complex structures. It was possible to fabricate other tissues or organs with this alginate-chitosan mixture.

Finally, hASCs were pluripotent cells. We verified that the hASCs that were seeded on the 3D printed AlCh PIC hydrogels adhered and proliferated, so the methods in this work could prepare hydrogels with a complex structure, to provide cells with appropriate growing environment. A further induced differentiation of hASCs in vitro could obtain neo tissue to repair or even replicate damaged tissues, thus the 3D printed AlCh PIC hydrogels had potential to be used for tissue engineering.

Supplementary Materials: The following are available online at http:/ / www.mdpi.com/2073-4360/10/6/664/s1, Figure S1: Compression modulus of bulk hydrogels during the degradation process.

Author Contributions: Q.L. (Qiongqiong Liu) conceived and designed the experiments and measurement. Q.L. (Qiongqiong Liu), Q.L. (Qingtao Li), and S.X. performed the experiments and measurement. Q.Z. and X.C. guided the experiments and measurement. All of the authors analyzed data.

Acknowledgments: This work was financially supported by the National Natural Science Foundation of China (Grant No. 21574045) and the Fundamental Research Funds for the Central Universities (Grant No. 2017ZD003).

Conflicts of Interest: The authors declare no conflict of interest.

\section{References}

1. Ribeiro, A.; Blokzijl, M.M.; Levato, R.; Visser, C.W.; Castilho, M.; Hennink, W.E.; Vermonden, T.; Malda, J. Assessing bioink shape fidelity to aid material development in 3D bioprinting. Biofabrication 2017, 10, 14102. [CrossRef] [PubMed]

2. Malda, J.; Visser, J.; Melchels, F.P.; Jüngst, T.; Hennink, W.E.; Dhert, W.J.A.; Groll, J.; Hutmacher, D.W. 25th anniversary article: Engineering hydrogels for biofabrication. Adv. Mater. 2013, 25, 5011-5028. [CrossRef] [PubMed]

3. Tirella, A.; Orsini, A.; Vozzi, G.; Ahluwalia, A. A phase diagram for microfabrication of geometrically controlled hydrogel scaffolds. Biofabrication 2009, 1. [CrossRef] [PubMed]

4. Sultan, S.; Siqueira, G.; Zimmermann, T.; Mathew, A.P. 3D printed of nano-cellulosic biomaterials for medical applications. Curr. Opin. Biomed. Eng. 2017, 2, 29-34. [CrossRef]

5. Leppiniemi, J.; Lahtinen, P.; Paajanen, A.; Mahlberg, R.; Metsä-Kortelainen, S.; Pinomaa, T.; Pajari, H.; Vikholm-Lundin, I.; Pursula, P.; Hytönen, V.P. 3D-Printable Bioactivated Nanocellulose-Alginate Hydrogels. ACS Appl. Mater. Interfaces 2017, 9, 21959-21970. [CrossRef] [PubMed]

6. Zhu, K.; Shin, S.R.; van Kempen, T.; Li, Y.C.; Ponraj, V.; Nasajpour, A.; Mandla, S.; Hu, N.; Liu, X.; Leijten, J.; et al. Gold Nanocomposite Bioink for Printing 3D Cardiac Constructs. Adv. Funct. Mater. 2017, 27. [CrossRef]

7. Domingues, R.M.A.; Gomes, M.E.; Reis, R.L. The Potential of Cellulose Nanocrystals in Tissue Engineering Strategies. Biomacromolecules 2014, 15, 2327-2346. [CrossRef] [PubMed]

8. Bidarra, S.J.; Barrias, C.C.; Granja, P.L. Injectable alginate hydrogels for cell delivery in tissue engineering. Acta Biomater. 2014, 10, 1646-1662. [CrossRef] [PubMed]

9. Pan, T.; Song, W.; Cao, X.; Wang, Y. 3D Bioplotting of Gelatin/Alginate Scaffolds for Tissue Engineering: Influence of Crosslinking Degree and Pore Architecture on Physicochemical Properties. J. Mater. Sci. Technol. 2016, 32, 889-900. [CrossRef] 
10. Bendtsen, S.T.; Quinnell, S.P.; Wei, M. Development of a novel alginate-polyvinyl alcohol-hydroxyapatite hydrogel for 3D bioprinting bone tissue engineered scaffolds. J. Biomed. Mater. Res. Part A 2017, 1457-1468. [CrossRef] [PubMed]

11. Farris, S.; Schaich, K.M.; Liu, L.S.; Piergiovanni, L.; Yam, K.L. Development of polyion-complex hydrogels as an alternative approach for the production of bio-based polymers for food packaging applications: A review. Trends Food Sci. Technol. 2009, 20, 316-332. [CrossRef]

12. Hong, J.; Kim, B.S.; Char, K.; Hammond, P.T. Inherent charge-shifting polyelectrolyte multilayer blends: A facile route for tunable protein release from surfaces. Biomacromolecules 2011, 12, 2975-2981. [CrossRef] [PubMed]

13. Luo, F.; Sun, T.L.; Nakajima, T.; Kurokawa, T.; Zhao, Y.; Sato, K.; Ihsan, A.B.; Li, X.; Guo, H.; Gong, J.P. Oppositely charged polyelectrolytes form tough, self-healing, and rebuildable hydrogels. Adv. Mater. 2015, 27, 2722-2727. [CrossRef] [PubMed]

14. Bhattarai, N.; Gunn, J.; Zhang, M. Chitosan-based hydrogels for controlled, localized drug delivery. Adv. Drug Deliv. Rev. 2010, 62, 83-99. [CrossRef] [PubMed]

15. Yu, X.; Qian, G.; Chen, S.; Xu, D.; Zhao, X.; Du, C. A tracheal scaffold of gelatin-chondroitin sulfate-hyaluronan-polyvinyl alcohol with orientated porous structure. Carbohydr. Polym. 2017, 159, 20-28. [CrossRef] [PubMed]

16. Li, Z.; Ramay, H.R.; Hauch, K.D.; Xiao, D.; Zhang, M. Chitosan-alginate hybrid scaffolds for bone tissue engineering. Biomaterials 2005, 26, 3919-3928. [CrossRef] [PubMed]

17. Baysal, K.; Aroguz, A.Z.; Adiguzel, Z.; Baysal, B.M. Chitosan/alginate crosslinked hydrogels: Preparation, characterization and application for cell growth purposes. Int. J. Biol. Macromol. 2013, 59, 342-348. [CrossRef] [PubMed]

18. Wang, G.; Wang, X.; Huang, L. Feasibility of chitosan-alginate (Chi-Alg) hydrogel used as scaffold for neural tissue engineering: A pilot study in vitro. Biotechnol. Equip. 2017, 31, 766-773. [CrossRef]

19. Zhai, X.; Ma, Y.; Hou, C.; Gao, F.; Zhang, Y.; Ruan, C.; Pan, H.; Lu, W.W.; Liu, W. 3D-Printed High Strength Bioactive Supramolecular Polymer/Clay Nanocomposite Hydrogel Scaffold for Bone Regeneration. ACS Biomater. Sci. Eng. 2017, 3, 1109-1118. [CrossRef]

20. Reed, S.; Wu, B.M. Biological and mechanical characterization of chitosan-alginate scaffolds for growth factor delivery and chondrogenesis. J. Biomed. Mater. Res. Part B Appl. Biomater. 2017, 105, 272-282. [CrossRef] [PubMed]

21. Chaudhuri, R.; Ramachandran, M.; Moharil, P.; Harumalani, M.; Jaiswal, A.K. Biomaterials and cells for cardiac tissue engineering: Current choices. Mater. Sci. Eng. C 2017, 79, 950-957. [CrossRef] [PubMed] 